\title{
What will the future hold: RNP quality control and degradation
}

\author{
KATRIN KARBSTEIN \\ Department of Cancer Biology, The Scripps Research Institute, Jupiter, Florida 33458, USA
}

Many will agree that the discovery of regulatory RNA molecules (miRNAs, piRNAs, IncRNAs, crRNAs, etc.) and the technological advances made possible through these basic scientific findings, are the biggest discoveries in the RNA world, and perhaps in the biosciences as a whole since the inception of the RNA journal. However, this kind of agreement is unlikely in regards to what the most important outstanding questions are. Perhaps these questions are unforeseeable, just like the discovery of entirely new classes of RNA molecules with diverse regulatory functions, conserved in all forms of life was unanticipated. Thus, I will instead discuss a problem I believe is interesting, exciting and important, without trying to claim that its solution will be game-changing: I believe that a critical open question in RNA biology is the elucidation of quality control and connected degradation pathways for nascent and damaged RNA-protein complexes.

There are many possible mechanisms for quality control and this word has been used in multiple different contexts. For the purpose of this article, quality control refers to functional tests of proper assembly and not just to mechanisms that ensure proper assembly or that prevent functionality of assembly intermediates.

Quality control is wide-spread and important in biology, with examples ranging from T-cell maturation (where the functionality of the T-cell-receptor is tested), over nonsense-mediated mRNA decay (which tests for correct splicing), to DNA replication (which ensures faithful WatsonCrick basepairing). The characterized systems ensure that newly made biomolecules are correctly assembled, or that damaged molecules are removed from circulation. While many of the described systems test the functionality of individual molecules, RNAs generally function as RNA-protein complexes (RNPs) with tRNAs perhaps being the most significant exception. Thus, quality control for RNA molecules generally involves testing of molecular assemblies.

RNPs are important regulators of gene expression at all levels: Telomerase ensures the integrity of the genome by maintaining the ends of our chromosomes; RITS and

\footnotetext{
Corresponding author: kkarbst@scripps.edu

Article and publication date are at http://www.rnajournal.org/cgi/doi/ 10.1261/rna.050658.115. Freely available online through the RNA Open Access option.
}

lncRNPs regulate chromatin structure; RISC regulates the stability and translatability of mRNAs; RNaseP, its cousin RNase MRP and snoRNPs are involved in the biogenesis of tRNAs and ribosomes; finally, spliceosomes and ribosomes are giant RNA-protein machines that allow for removal of introns and translation of proteins from the resulting mRNAs.

Generally, the RNAs within these machines are transcribed as precursor molecules and then processed and assembled with their proteinaceous binding partners. Binding partners can number between just one (bacterial RNase P) and $>100$ (the spliceosome). Assembly often occurs in a cellular compartment different from where the RNP functions. For example, ribosomes are largely assembled in the nucleus but function in the cytoplasm, while snRNPs are assembled with Sm proteins in the cytoplasm but operate in the nucleus. In both cases final maturation steps occur in the cellular compartment where the RNP functions. Presumably, this separation of assembly and function prevents assembly intermediates from carrying out partial functions, or from blocking the activity of mature RNPs. Generally speaking, the assembly process for RNPs is highly hierarchical. Hierarchical assembly pathways provide for internal quality control systems, as assembly of late components proceeds only when prior components are all present. Early intermediates are probably highly susceptible to degradation, while later intermediates are stabilized, and perhaps require targeting to degradation machineries, such as the exosome, if assembly fails.

Work on the assembly of many of these RNPs has provided surprising and interesting insights. For example, the R2TP (Rvb1-Rvb2-Tah1-Pih1) complex, which contains two AAA+-type ATPases and two adaptors, serves as a chaperone for assembly of many divergent RNPs, as well as protein complexes, by modulating the activity of Hsp90.

What is not well documented in the literature are functional tests of the newly-assembled RNPs. Nevertheless, misassembly of RNPs underlies several important human diseases, including the so-called ribosomopathies, a diverse class of diseases all causally linked to defects in the assembly of ribosomes. Likewise, defects in the assembly of other RNPs

(C) 2015 Karbstein This article, published in $R N A$, is available under a Creative Commons License (Attribution-NonCommercial 4.0 International), as described at http://creativecommons.org/licenses/by-nc/4.0/. 
leads to severe human diseases. For example, spinal muscular atrophy (SMA) is caused by defects in the assembly of Sm proteins onto snRNAs and dyskeratosis congenita is caused by defective telomerase assembly; in addition, Hsp90 inhibitors, which block the assembly of many RNPs (as well as other protein complexes), are promising anti-cancer drugs. These diseases demonstrate the importance of quality control in the assembly of RNPs.

tRNAs, which are not assembled in an RNP, but nevertheless processed and extensively modified before being released into the translating pool, were shown to undergo just such a functional test, as successful export requires the ability to charge the tRNA in a pioneering round in the nucleus. Similarly, recent work on the assembly of the two ribosomal subunits has revealed that translation-like mechanisms test the ability of nascent small subunits to bind the large subunits as well as certain translation factors. Similarly, nascent large subunits are tested for their ability to bind a translation-factor-like cofactor, which carries out a translocation-like movement to release not a tRNA, but an assembly factor. How exactly failure to pass quality control leads to degradation remains unknown, and may simply occur by kinetic competition. Despite these recent insights, there are still no mechanisms described that test the ribosome's ability to correctly decode an mRNA, form a peptide bond, or translocate by exactly three nucleotides in a GTP-dependent manner. Yet, during translation these processes are so carefully regulated and themselves proofread, it is hard to imagine ribosomes being released into the translating pool, without testing them for this ability.

Similarly, spliceosomes carry out perhaps an even more difficult task: finding splice sites, which are poorly defined at the sequence level, and then excising introns precisely and correctly every time, except when they are not supposed to because of regulated alternative splicing. As described above, the importance of correct spliceosome assembly is reflected in diseases such as SMA. Yet, there is virtually no information about functional tests of spliceosome assembly. What could such functional tests examine? The possibilities range from the formation of snRNP-snRNP complexes (like perhaps the penta-snRNP) to the ability to recognize splice sites, as well as the exceptional dynamics of the system. Finally, telomerase, RITS, RISC and CRISPR RNPs have important functions in genome maintenance or gene ex- pression. It is hard to imagine that their highly-regulated destructive potential would not be tested.

Interestingly, the quality control checks for nascent ribosomal subunits occur in the cytoplasm, where ribosomes normally function, and not in the nucleus, where most of assembly takes place. Of course this is because the translational machinery is located in the cytoplasm, and used for quality control in this case. In that context, it is interesting to note that also for spliceosomes and other RNPs, final maturation steps are carried out in the cellular compartment where they function, even though most of assembly takes place elsewhere. Perhaps this is a hint at the existence of such quality control pathways, which similarly take advantage of the existing functional machinery.

In addition to quality checks of nascent RNPs, all functional RNAs are likely surveyed throughout their life-time to ensure that damaged RNPs are removed from the cell. This is well documented for mRNAs, as well as non-functional ribosomes. Since both defects in the mRNA or the ribosome will lead to nearly identical stalled intermediates, it is not surprising that both mRNA and ribosome surveillance is jump-started by Hbs1-dependent delivery of Dom34 to the stalled ribosome in a process that resembles termination. Nevertheless, how degradation of both the mRNA and their bound ribosomes proceeds (beyond initiating disassembly of the translating ribosomes from the mRNA, and the involvement of a yet-to-be discovered endonuclease, which cleaves the mRNA) remains poorly understood. This is especially the case for the ribosomes.

Generally, quality control pathways require not just the identification of defective or non-functional RNPs (via quality control mechanisms), but also that these be marked, perhaps by posttranslational modification (PTM) of the proteins, by polyadenylation of the RNA, or by endonucleolytic RNA cleavage. PTM marks could then help target the defective RNPs to the proteasome or to the autophagy pathway, which can target ribosomes specifically in a process called ribophagy. Similarly, nuclear polyadenylation provides a targeting signal for the exosome. Nevertheless, which, if any, of these machineries are involved in RNP degradation, how proteins are removed from the RNA, and if there is recycling of protein components remains entirely unexplored, for the next 20 years of RNA research. 

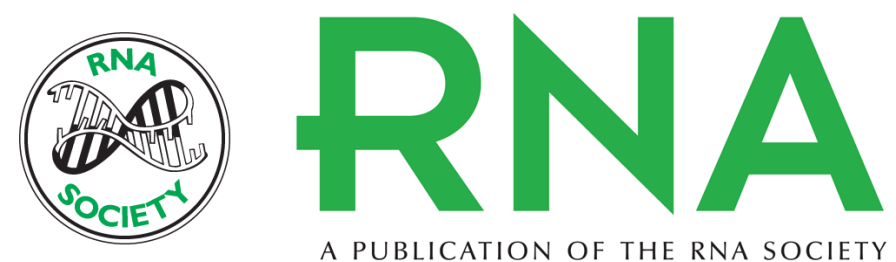

A PUBLICATION OF THE RNA SOCIETY

\section{What will the future hold: RNP quality control and degradation}

Katrin Karbstein

RNA 2015 21: 657-658

Open Access Freely available online through the RNA Open Access option.

Creative This article, published in RNA, is available under a Creative Commons License Commons (Attribution-NonCommercial 4.0 International), as described at License http://creativecommons.org/licenses/by-nc/4.0/.

Email Alerting Receive free email alerts when new articles cite this article - sign up in the box at the Service top right corner of the article or click here. 